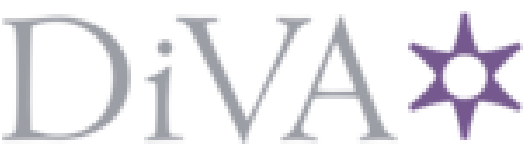

http://www.diva-portal.org

\title{
Postprint
}

This is the accepted version of a paper published in European Journal of Marketing. This paper has been peer-reviewed but does not include the final publisher proof-corrections or journal pagination.

Citation for the original published paper (version of record):

Paschen, J., Wilson, M., Robson, K. (2020)

\#BuyNothingDay: investigating consumer restraint using hybrid content analysis of Twitter data

European Journal of Marketing, (2): 327-350

Access to the published version may require subscription.

N.B. When citing this work, cite the original published paper.

Permanent link to this version:

http://urn.kb.se/resolve?urn=urn:nbn:se:kth:diva-268852 


\section{\#BuyNothingDay: investigating consumer restraint using hybrid content analysis of Twitter data}

\section{Abstract}

\section{Purpose:}

This study aims to investigate motivations and human values of everyday consumers who participate in the annual day of consumption restraint known as Buy Nothing Day (BND). In addition, this study demonstrates a hybrid content analysis method in which artificial intelligence and human contributions are used in the data analysis.

\section{Design/methodology/approach:}

This research uses a hybrid method of content analysis of a large Twitter data set spanning three years.

\section{Findings:}

Consumer motivations are categorized as relating to consumerism, personal welfare, wastefulness, environment, inequality, anti-capitalism, financial responsibility, financial necessity, health, ethics and resistance to American culture. Of these, consumerism and personal welfare are the most common. Moreover, human values related to "openness to change" and "self-transcendence" were prominent in the BND tweets.

\section{Research limitations/implications:}

This research demonstrates the effectiveness of a hybrid content analysis methodology and uncovers the motivations and human values that average consumers (as opposed to consumer activists) have to restrain their consumption. This research also provides insight for firms wishing to better understand and respond to consumption restraint.

\section{Practical implications:}

This research provides insight for firms wishing to better understand and respond to consumption restraint. 


\section{Originality/value:}

The question of why everyday consumers engage in consumption restraint has received little attention in the scholarly discourse; this research provides insight into "everyday" consumer motivations for engaging in restraint using a hybrid content analysis of a large data set spanning over three years.

\section{Keywords:}

Content analysis, Anti-consumption, Consumerism, BlackFriday, BuyNothingDay, Consumer restraint, Hybrid method, Twitter data

\section{Paper type:}

Research paper 


\section{Introduction}

Consumers today show record levels of spending: the ratio of household debt to disposable income reached almost 128 per cent in 2017 (OECD, 2019). In light of this spending boom, it is not surprising that consumer research focuses on how and why consumers satisfy needs and wants through market offerings. Yet, consumption restraint - the purposeful practice of restricting consumption - is an increasingly popular movement among consumers and has received increasing attention in academic literature as a result (Balderjahn et al., 2018; Cronin et al., 2015; Galvagno, 2011; Lee et al., 2011; Odou and de Pechpeyrou, 2011; Pentina and Amos, 2011).

Until recently, experiences and efforts around consumption restraint were largely shared among like-minded people, such as anti-consumption groups, and have been explored by researchers with specific focus on these populations. As a result, the existing literature has studied consumption restraint with self-identified anti-consumers or activists (Black and Cherrier, 2010; Cherrier et al., 2011; Garcia-Bardidia et al., 2011; Pentina and Amos, 2011). Yet, consumption restraint is growing in popularity among typical consumers as well, bolstered by cultural, environmental and economic shifts such as increasing concerns about the natural environment and climate change (Black and Cherrier, 2010; Dobscha, 1998). For example, following the global economic crises in 2008 and 2009, there has been a renewed interest in the notions of austerity and frugality as opportunities for achieving sustainable consumption (Evans, 2011). Yet, little is currently known about motivations of the broader, general population who may not identify as anti-consumers but who engage in consumption restraint, nevertheless. As such, existing literature portrays an incomplete (Locke and GoldenBiddle, 1997) picture of the consumers who engage in this practice.

As typical consumers become more aware of issues related to materialism, minimalism and the environmental impact of their consumption, understanding the motivations and behavior of the broader consumer is a necessary and timely undertaking. Specifically, an enhanced understanding of the motivations for consumption restraint is important for practitioners and scholars. First, for practitioners, it provides insight into consumer motivations that managers can use to craft strategies and appropriate responses to typical consumers who wish 
to practice some level of restraint. Second, for scholars, it is relevant as it adds depth to the growing literature on consumption restraint by providing understanding of mainstream restraint. Research is needed to understand how a typical consumer (as opposed to how consumers whose self-identities and lifestyles rely heavily on non-consumption practices) restrains his/her consumption.

In addition to the growing popularity of the consumption restraint phenomena, the rise of social media brings the narratives of typical consumers to the fore, with the potential to increase their reach and impact. For instance, communications on social media sites have been credited for mobilizing significant social events such as the "Arab Spring" (Brown et al., 2012) or the Stanley Cup Riots in Canada in 2011 (Stueck, 2018). Moreover, content shared on Twitter has been found to impact audience opinions and attitudes toward organizations and brands (Burch et al., 2015; Jin and Pua, 2014; Qin, 2015). These phenomena suggest that it is worthwhile and important for firms to gain a broader understanding of the "how" and "why" of consumption restraint behavior from social media communications. In addition, social media content is spontaneous, easily accessible, live (Tirunillai and Tellis, 2014) and based on a large number of contributions from many individuals. Thus, communications on social media sites offer valuable opportunities for marketers to create knowledge about the creators of these data, including about their motivations, wants, needs, values and behaviors.

In this paper, we explore consumption restraint through exploration of tweets about "Buy Nothing Day" (BND). In BND, consumers commit to buying nothing on the most popular shopping holiday of the year: "Black Friday". We explore consumption restraint through BND participation via content analysis of user-generated comments over three years on Twitter. Our study uses a hybrid content analysis, combining computerized analysis via an artificial intelligence (AI) application and human coding. In the extant literature, hybrid content analysis has been discussed as an effective approach to generate insights from unstructured text data (Lewis $e t$ al., 2013; Su et al., 2017; Zamith and Lewis, 2015). Our study demonstrates such a hybrid content analysis method. 
The research questions that our paper aims to answer are:

RQ1. What motivation(s) does the general consumer population have to participate in consumption restraint through BND?

RQ2. How can different content analysis methods-manual and automated using AI- be used to extract insights from user-generated data?

In answering these questions, this research makes two key contributions to literature. First, this research contributes to our current understanding of consumption restraint in consumers through an analysis of consumer tweets related to BND. More specifically, this study extends the previous literature on consumption restraint by identifying new motivations for why consumers partake in consumption restraint. Second, this research contributes to an understanding of the methodological approaches that can be used to gain market intelligence from unstructured data using human and AI methods. This latter point has been identified as an area under-represented in the current literature and requiring further scholarly attention (Balducci and Marinova, 2018; Duan et al., 2019; Gupta et al., 2018; Jarrahi, 2018; Kaplan and Haenlein, 2019; Singh et al., 2019). In what follows, we review the literature on consumption restraint and provide a background on BND, followed by a literature review on content analysis methods. Subsequently, we describe our research methodology and the results of our hybrid content analysis in detail. We conclude our article with a summary of contributions, limitations and suggestions for future research.

\section{Literature review}

\section{Consumption restraint, consumer resistance and anti- consumption}

Consumption restraint refers to consumer resistance against the culture of consumption (Penaloza and Price, 1993) and includes anticonsumption and consumer resistance. In this section, we explain these constructs, including the differences and relationships between them. Specifically, using the framework suggested by Füller (2010), we describe 
the primary actor(s) (the who?), the specific activities (the how?) and the object (the what?) for each, anti- consumption and consumer resistance, thus providing a comprehensive understanding of the constructs that guide our research (Table I).

Table I Key terms within consumption restraint: anti- consumption and consumer resistance

\begin{tabular}{|c|c|c|c|}
\hline Key Term & $\begin{array}{l}\text { Actors (the } \\
\text { who?) }\end{array}$ & Behavior (the how?) & Object (the what?) \\
\hline $\begin{array}{l}\text { Anti- } \\
\text { consumption }\end{array}$ & Individuals & $\begin{array}{l}\text { Any or all of: } \\
\text { - Rejecting: intentionally } \\
\text { excluding offerings from } \\
\text { consumption (Lee et al., } \\
\text { 2011). } \\
\text { - Restricting: reducing or } \\
\text { limiting consumption of } \\
\text { offerings, often for a specific } \\
\text { period of time (Eagle and } \\
\text { Dahl, 2015; Lee et al., 2011). } \\
\text { - Reclaiming: shifting the } \\
\text { acquisition, use and dis- } \\
\text { possession of offerings (Lee } \\
\text { et al., 2011) }\end{array}$ & $\begin{array}{l}\text { Process of consumption, } \\
\text { i.e. the acquisition, use } \\
\text { and disposition of } \\
\text { commodified goods, } \\
\text { including ideas, services, } \\
\text { products, brands and } \\
\text { experiences (Cherrier et } \\
\text { al., 2011; Lee et al., 2011) }\end{array}$ \\
\hline $\begin{array}{l}\text { Consumer } \\
\text { resistance }\end{array}$ & Individuals & $\begin{array}{l}\text { Resisting power influences } \\
\text { attempted by firms or other } \\
\text { consumers over an individual } \\
\text { (Lee et al., 2011; Roux, 2007) }\end{array}$ & $\begin{array}{l}\text { Perceived dominant force } \\
\text { exerted by certain actors, } \\
\text { behaviors, and devices } \\
\text { (Cherrier, 2009; Cherrier } \\
\text { et al., 2011; Roux, 2007) }\end{array}$ \\
\hline
\end{tabular}

Anti-consumption encompasses individuals' behaviors that are in opposition to the process of consumption (Cherrier et al., 2011; Lee et al., 2011, 2009; Zavestoski, 2002). For example, anti-consumption can involve avoidance of consumption or resentment toward consumption of any commodified offering, including products, services, brands or ideas (Zavestoski, 2002). In the extant literature, anti-consumption is broadly classified into a phenomenon encompassing three, non-exclusive behaviors: reject, restrict and reclaim (Lee et al., 2011). Reject behavior includes individuals intentionally excluding particular offerings from their consumption behavior. In the process of restricting, individuals abstain from, reduce or limit consumption of certain products, often for a specific period of time (Eagle and Dahl, 2015; Lee et al., 2011). Limiting consumption is often linked to political or social movements aimed at temperance of consumption. Reclaim behavior represents an ideological shift regarding the behaviors of acquisition, use and dispossession of offerings (Lee et al., 2011). For instance, voluntary 
simplifiers opt to consume only in a matter that will improve their happiness, and thereby do not consume otherwise (Craig-Lees and Hill, 2002). In this regard, voluntary simplifiers' behavior reflects their ideological stance (Lee et al., 2011), for example when deciding to grow their own vegetables rather than acquiring them through businesses.

Consumer resistance focuses on individuals opposing a leading force exerted by certain actors, such as businesses or other consumers, behaviors or devices (Cherrier, 2009; Lee et al., 2011; Roux, 2007). Specifically, as firms attempt to influence consumers' behaviors, for example via advertising or selling tactics, they aim to exert power over consumers. Consumers can resist these influences, for example by expressing discontent in various forms. As an illustration, consumers can resist firms' selling tactics for reasons of autonomy or ethics. This behavior can be directed toward specific brands (Hogg et al., 2009), corporate greed or the dominant system of capitalism in general. Complaining behavior, boycotts and "culture jamming" are all expressions of discontent, whereby the latter encompasses behavior intended to combat consumerism through disrupting mainstream consumption or corporate advertising (Kozinets and Handelman, 2004). Some consumers also choose to avoid companies as an exchange partner in consumption, instead favoring alternative channels, such as consumerto-consumer exchanges, sharing or second-hand markets (Lee et al., 2011).

The above discussion highlights that anti-consumption and consumer resistance are distinct, but not mutually exclusive concepts, and that they both fall under the larger umbrella of research on consumption restraint. In some cases, consumer resistance is manifest by anti-consumption behavior, for example through boycotts, or the rejection of certain products, brands or firms (Lee et al., 2011).

However, consumer resistance may also be expressed through certain consumption behaviors, for example, through buying used or sharing access to offerings (Albinsson et al., 2010). In the same way, not all facets of anti- consumption behavior need to necessarily involve consumer resistance against attempted influences by businesses or other economic actors. This suggests that anti-consumption and consumer resistance, while different, overlap in parts (Figure 1).

To summarize, anti-consumption behavior is concerned with the behaviors of rejecting, restricting and reclaiming of commodified offerings, while consumer resistance focuses on consumers opposing 
offerings associated with a structure of dominance. "Anticonsumption is concerned with consumption issues while consumer resistance is concerned with power issues" (Lee et al., 2011). The research presented in this paper is positioned at the intersection of the domains, as illustrated in Figure 1 below.

Figure 1 Delineating BND participation within the domains of consumption restraint (anti- consumption and consumer resistance)

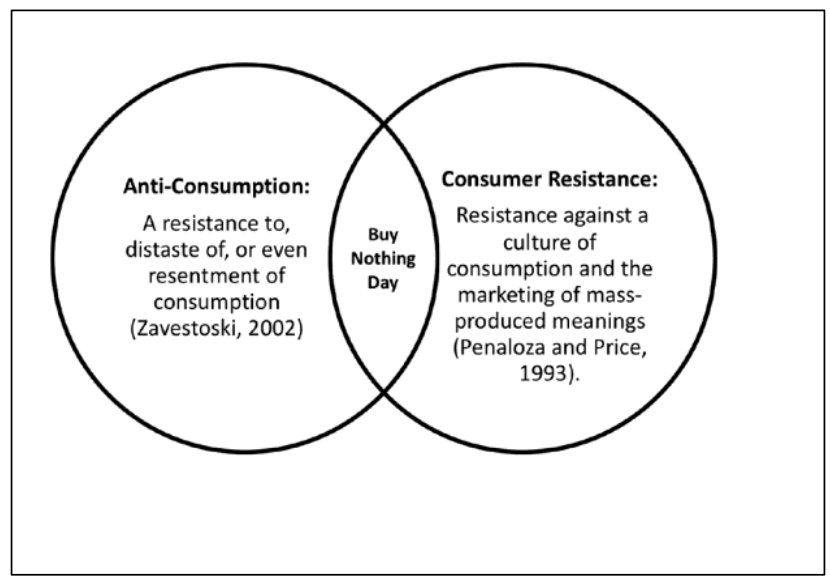

\section{Consumption restraint, internal motivations and values}

Recent work has posited that in any form, consumption restraint practices are rooted in consumer subjectivity (Cherrier et al., 2011). Thus, a practical view of consumption restraint adopts a micro-perspective in which consumption restraint behavior is related to and impacted by an individual's self-identity: "[...] anti-consumption, like consumption practices, enable consumers to express their values, ideas, beliefs and overall identities" (Cherrier et al., 2011, p. 1,758). This micro-perspective is echoed by other scholars who suggest that consumption restraint practices are affected by psychological factors (Pepper et al., 2009), such as the self-consciousness, self-actualization and assertiveness of the individual (Iyer and Muncy, 2009; Lee et al., 2011). As such, personal narratives, values, culture, experience and perception inform an individual's decision to restrain their consumption. 
The extant literature offers various perspectives on the root causes for consumption restraint behaviors within the micro-perspective and one's self-identity. Iyer and Muncy (2009), for instance, classify anticonsumption behavior based on two dimensions. The first dimension is the purpose of anti-consumption, which the authors categorize as either for personal or social purposes. The second dimension is the degree of specificity regarding the object of anti-consumption which is divided into specific products, brands or offerings on the one hand, and general consumption resistance on the other hand. The typology suggested by Iyer and Muncy (2009) with four types of anti-consumers has been useful in characterizing certain forms of consumption temperance in a number of scholarly studies (Galvagno, 2011; Odou and De Pechpeyrou, 2011; Pentina and Amos, 2011; Rindell et al., 2014).

Another study within the micro-perspective explores self-identity with two consumer restraint groups - culture jammers and voluntary simplifiers (Cherrier, 2009). The results from in-depth interviews in this study suggest that individuals self-identify either as a hero (whose goal is to change the world) or as someone who is a project resistor (whose goal is to change themselves). Cherrier et al. (2011), using in-depth interviews, found that non- consumption was typically associated with two themes: first, a desire to be unlike a typical consumer or "consumer resistance against 'them' (other careless consumers)" (Cherrier et al., 2011, p. 1,764). The second theme is the adoption of mundane, everyday practices that support sustainability within the context of consumers' distinct selfconcepts, individualities and everyday circumstances (Cherrier et al., 2011). Research on consumption restraint, including anti-consumption and consumer resistance, reveals that just as what we buy says something about us, so too can our non-consumption convey aspects of our identity (Cherrier and Murray, 2007).

In addition, a substantial body of research has related an individual's value system to the internal motivations for and the behavioral manifestation of consumption restraint practices. Human values, defined as enduring beliefs that pertain to desirable end states or behaviors that transcend specific situations, guide a person's behavior and the evaluation thereof (Schwartz and Bilsky, 1987). Extant studies have examined the relationship between human values and various forms of consumption restraint behavior (Jägel et al., 2012; Pepper et al., 2009), and among the most widely used and validated models is Schwartz' value framework. Schwartz' $(1992,2012)$ model specifies ten motivational 
values and four higher-order value orientations that are organized along two bi-polar dimensions: self-enhancement vs selftranscendence (Dimension 1) and openness to change vs conservation (Dimension 2). The first value orientation, "self-enhancement," includes power and achievement values, thereby highlighting selfinterest, while, the second, "self-transcendence," emphasizes concern for others. The third value orientation, "openness to change" highlights independent action and thought, whereas, fourth, "conservation" is characterized by self-restriction and resistance to change (Schwartz, 1992, 2012).

Schwartz' value framework has provided valuable contributions to the literature on various forms of consumption restraint. Pepper et al. (2009), for instance, examine the relationship between Schwartz' values and consumption behavior among self-identified frugal consumers. The results from mail-questionnaires suggest that frugal purchasing behavior is positively related to the values "conformity" and "tradition" and thus positively related to the value dimension "conservation" (Pepper et al., 2009). In addition, the results suggest that "power" and "hedonism" which are both values within the dimension of "self- enhancement" are negatively correlated to frugal behavior. Shaw et al. (2005) explore the prominence of Schwartz' values pertinent to ethical consumption decisions with self- perceived ethical consumers using focus groups. Jägel et al. (2012) study consumption behavior among ethical clothing consumers and how these are linked back to one's values, while Gilg et al. (2005) use Schwartz' value model to provide insight into environmentally friendly consumption practices. Thus, in the literature, human values are discussed as an important construct that relate to and may impact consumption restraint practices.

The body of literature on consumption restraint has generally focused on understanding the motivations and behavior of selfidentified resistors and consumer activists (Black and Cherrier, 2010; Cherrier et al., 2011; Garcia-Bardidia et al., 2011; Pentina and Amos, 2011). However, consumers who self-identify as members of groups that restrict consumption are atypical and represent a small fraction of the market. Less light has been shed on the more typical consumer specifically those consumers who do not self-identify as culture jammers, voluntarily restrainers or as members of other specific groups which postulate restrictive consumption norms and practices. 
As a result, the existing literature on consumption restraint portrays an incomplete picture of consumers (Locke and Golden- Biddle, 1997) who engage in this practice.

Moreover, the understanding of one's motivations and values as it relates to consumption restraint practices has primarily been derived in studies using surveys, in-depth interviews or focus groups. While these studies have provided valuable contributions to the body of knowledge, there are methodological limitations related to the use of questionnaires. One such limitation relates to self-awareness: individuals may not know what their motivations or values are (Cheng et al., 2012). In addition, the use of questionnaires raises concerns of self- report biases and socially desired responses. Moreover, to assess human values, Schwartz' value model is operationalized by a battery of 56 or 57 individual values contained in the Schwartz value survey (Pepper et al., 2009) which can be cumbersome for respondents to complete. Because of these limitations, it is problematic to rely entirely on questionnaires when studying consumption restraint. As such, there are benefits to examining an existing corpus of data on consumption restraint, generated by consumers themselves.

The aim of this research is to explore individuals' motivations and values that lie at the core of consumption restraint among typical consumers, as opposed to self-identified sub-groups. This area of the consumption restraint phenomenon has previously been underrepresented in the literature. Addressing this gap is important for both research and practice. For researchers, it extends the body of knowledge on consumption restraint, and specifically with respect to consumers' self-identity, motivations and values. For managers, it provides insight into the motivations of consumer behavior, specifically why typical consumers engage in consumption restraint behavior. Knowledge about consumer motivations is an important form of market knowledge (Kohli and Jaworski, 1990) that is critical for managers in designing and executing effective marketing strategies and tactics. In addition, and as previously outlined, user- generated communications on social media can affect other individuals' opinions and behaviors. In addition, within the body of research on consumption restraint, to the best of our knowledge, there are no studies that examine consumer motivations specifically related to BND. We explain BND in more detail in the following section. 


\section{Buy Nothing Day and Black Friday}

"Black Friday" is the day after the American celebration of Thanksgiving in which stores have some of their biggest sales promotions of the year. In 2005, the Monday after Thanksgiving was coined as "Cyber Monday" in an attempt to encourage shoppers to engage in online shopping. Black Friday is currently the most popular day to shop in America across all age groups. In 2015, 154 million Americans went shopping in stores over the Black Friday weekend, and 122 million Americans engaged in online shopping on Cyber Monday (Reynolds and Smith, 2016). Despite the fact that Black Friday originated in the USA, the shopping holiday has expanded around the world (Black Friday's travels around the world, 2015).

In stark contrast to the spending and shopping that occurs on Black Friday, BND participants pledge to buy nothing for $24 \mathrm{~h}$ rather than partake in the consumption frenzy (Britannica Academic, 2020). Thus, BND is an annual day of consumer resistance which coincides with "Black Friday". As Black Friday has expanded internationally, so has the BND movement, which now has participants in North America, Asia, Europe, South America and Australia.

\section{Content analysis}

In the age of big data, consumers produce an enormous volume of data 2.5 quintillion bytes every day in 2018 (Marr, 2018), and the data volume has been growing at an exponential pace driven by the rise of the Internet of Things (Robson et al., 2016) and the increasing use of social media platforms (Kietzmann et al., 2011). On Facebook, for example, every $60 \mathrm{~s}$, users post 510,000 comments and update 293,000 statuses; on Twitter, users share close to 475,00o tweets a minute (Domo, 2018). This usergenerated content created in real-time can provide valuable insights into people's motivations, wants, values and day-to- day activities.

Content analysis, a technique to organize and make sense of the words, phrases and language as reflected in the narrative texts (Short et al., 2018) has long been used as a methodology in marketing and a range of other scientific fields (Kassarjian, 1977). Traditionally, content analysis has been performed manually by trained researchers. 
Today, methods of interpreting and extracting meaning from written text via computerized content analysis are becoming increasingly sophisticated (Karpf, 2012; Lewis et al., 2013) and are considered to be a reliable and highly efficient methods of data analysis (Lacy et al., 2015; Short et al., 2009, 2018). Computerized content analysis has provided valuable insight into understanding user-generated textual data (Dabirian et al., 2017; Lewis et al., 2013; Paschen et al., 2017; Pitt et al., 2017; Su et al., 2017), firm-generated communication (Pitt et al., 2018a, 2018b) and content generated by third-party organizations, such as media outlets (Paschen, 2019) or corporate referents (Short and Palmer, 2003). Computerized content analysis is touted as a highly valuable methodological approach within consumer research in general (Humphreys and Wang, 2017). In addition, computerized content analysis has been used to investigate consumer restraint (Hoffmann, 2011).

Content analysis can be used to investigate data from a wide range of sources. In particular, social media offers a wealth of readily accessible data for consumer behavior research, such as consumer attitudes, opinions and experiences (Kietzmann et al.,2011; Van Laer et al., 2018). Unsurprisingly, many have turned to social media to obtain large amounts of data for content analysis (Avery et al., 2010; Lai and To, 2015; Su et al., 2017).

In particular, many researchers have used Twitter as the source of their data (Humphreys et al., 2014; Qin, 2015; Su et al., 2017). Asur and Huberman (2010) used Twitter data to uncover information regarding consumer demand which could be used to forecast movie earnings. In addition, Twitter data has been used in research with applications in forecasting online book sales, as well as fluctuations in the stock market (Da et al., 2011; Goel et al., 2010; Gruhl et al., 2005). Indeed, Twitter is thought to be a source of highly valuable data for two key reasons. First, the default privacy setting on Twitter is "public," which means that all tweets from users who have not specifically changed their account settings to "private" are searchable on Twitter, on Google, and are archived in the Library of Congress (Humphreys et al., 2014; Raymond, 2010). Research has also found that default privacy settings on social media are highly influential as most users do not change these settings because they either do not know how or do not know that it is possible (Shah and Kesan, 2003). More recently, Moore (2009) found that in 2009 less than 8 per cent of new Twitter users change the default privacy 
setting. Second, the use of hashtags on Twitter is a highly useful way of searching for relevant data. Not only do hashtags in tweets allow individuals from different networks to connect on similar topics, but they also allow researchers to identify connections between these different individuals and that hashtag (Humphreys et al., 2014).

Despite the clear value of using computerized content analysis as a methodology, a number of researchers have noted limitations and issues with automated content analysis, such as issues related to validity (Grimmer and Stewart, 2013) and to extracting nuanced or subtle meanings from complex language (Conway, 2006; Lewis et al., 2013). These issues can be overcome with traditional, manual content analysis - yet manual content analysis brings its own limitations notably that it is generally more costly than automated content analysis (Lacy et al., 2015) and that humans are limited in their ability to analyze large databases in a timely fashion (Su et al., 2017). The trade-offs between automated and manual content analysis are clear, leading many to suggest that when possible, a hybrid approach is preferable (Lewis et al., 2013; Su et al., 2017; Zamith and Lewis, 2015) and with Conway (2006) going as far as to suggest that it is the "gold standard" for research using content analysis.

In this research, we use a Twitter data set spanning over three years to understand consumer restraint on BND. This data set is analyzed using the hybrid method, which involved a computerized content analysis using the AI application IBM Watson as well as manual classification and coding by trained researchers. The following section details the methods of our hybrid content analysis of Twitter data.

\section{Method}

In this study, we investigate the motivations for participating in BND and the values toward BND as expressed in user-generated tweets about this event. In this section, we present the methods of our research. 


\section{Sample}

To assess the general public's motivations for participating in BND, Twitter data was obtained during the Black Friday shopping seasons in 2016, 2017 and 2018. The data set for each year included all tweets containing the term "Buy Nothing Day" or the hashtag "\#BuyNothingDay" within a 10-day time period surrounding "Buy Nothing Day" (November 24th in 2016; 23rd in 2017 and 22nd in 2018). A 10-day time period was chosen to include the related shopping holiday Cyber Monday and any pre-BND anticipation and post- BND reflection. Although the time period covered the span of 10 days, each year roughly 70 per cent of the tweets were sent on BND itself.

The full data set of tweets containing the term "Buy Nothing Day" or \#BuyNothingDay was cleaned to remove duplicate tweets (i.e. retweets). In addition, as this study focuses on individuals' motivations, all tweets originating from a corporate account, a brand or an organization were eliminated. Finally, we removed all non-English tweets because of the language limitations of the authors. Ultimately, this led to a final data set of 5,603 tweets across three years. In what follows, we describe our hybrid content analysis of these tweets, which involved manual classification and coding as well as automated content analysis using the AI application IBM Watson.

\section{Manual classification and coding}

Prior to analyzing the Twitter data, we conducted an extensive literature review on consumer behavior related to consumption restraint to help inform our analysis. From this review, a list of initial categories was created to capture the potential motivations of consumers participating in BND. In particular, we began with Iyer and Muncy's (2009) work that categorized the purpose of anti-consumption behavior as either societal or personal. To add further specificity, we continued to read the wider literature to better understand possible personal or societal reasons.

We modeled our manual content analysis methodology based on that of Robson et al. (2015) which encompassed three steps. In the first step, two researchers independently read 150 randomly selected tweets and 
coded these tweets into the categories of motivations for participating in BND developed from the literature review. In Step 2, the researchers reviewed each tweet together and deliberated each coding decision until consensus was reached. In the third step, the researchers discussed and compared the coding categories that were identified and extracted commonalities between them. From these commonalities the list of initial coding categories was revised, and associated definitions and examples were developed for each coding category of motivation. All of the remaining tweets were then coded using the coding categories developed in Step 3. This procedure was repeated for each year's data set. In 2017 and 2018, new categories of consumer motivations emerged and were added to the coding categories. A random selection of 150 tweets from the previous years' tweets was re-evaluated to determine if these new codes had been present (but overlooked) in coding. No such evidence was found. Across all three years, tweets could display more than one motivation and were therefore coded as such.

\section{Motivations for Buy Nothing Day participation}

From the coding procedure described in the previous section, 11 categories of motivations emerged from the manual content analysis: consumerism, personal welfare, wastefulness, environmental, inequality, anti-capitalism, financial responsibility, financial necessity, health, ethics and resistance to American culture. In addition to these categories, tweets were coded as "no-motivation" if they contained no apparent motivation. To refine understanding of these "no-motivation tweets," the valence of each tweet was recorded based on whether the sentiment toward BND was positive, negative or neutral. As such, these tweets were coded as either no motivation-positive, no motivation-negative or no motivationneutral. Thus, across the three years, we uncovered 11 major classifications of motivations and 3 classifications for tweets revealing no motivations at all. These categories are described below. 
Consumerism. Consumerism is a preoccupation with and an inclination toward the buying of goods and services in ever increasing amounts and is often associated as a social force that is dominant in today's culture (James and Szeman, 2010). Thus, the consumerism category encompasses the madness of Black Friday (shopping frenzy, fighting over deals, overcrowded and chaotic shopping malls), materialism (fighting the urge to consume more, the pressure or desire to seek happiness through purchases and mindless consumption) and commercializing the holidays. The tweet "\#blackfriday epitomises all that's wrong with of our consumerist lifestyles \#buynothingday \#boycottblackfriday" is an example of a tweet expressing motivation related to consumerism.

Personal welfare. Many consumers see Black Friday as a distraction from the more important things in life. Thus, the personal welfare category was created to classify tweets pertaining to shopping less and living more, opting to go outside, to spend more time with friends and loved ones, or to engage in other activities or experiences not related to shopping. This is reflected in the following tweet: "Make something, see something, talk to someone, sit, think, read, listen to music, go for a walk, [...]\#buynothingday". In addition, the personal welfare category included religious considerations, such as consumption temperance because of observance of the Sabbath which is exhibited in the following tweet: "As for \#buynothingday, you know I had one of these weekly for several years? Didn't even carry money. It was part of my Sabbath observance".

Wastefulness. "Wastefulness" included themes associated with wasting things, buying "crap" and questioning whether consumers actually need the items that they purchase. As such, tweets that mentioned making purchases count, and avoiding buying things that are not needed and would likely be thrown out were categorized as wasteful. For example, the following tweet reveals a motivation related to the perceived wastefulness of Black Friday and exemplifies this classification: "Think twice before you buy something! Today's trends are tomorrow's trash \#BlackFriday is \#BuyNothingDay".

Environmental concerns. Tweets that referenced issues related to pollution, climate change, limited natural resources, mother earth and other impacts on the planet were categorized as indicative of motivations pertaining to environmental concerns. For example, the following tweet was coded as displaying the environmental motivation: "I'm committed to 
\#BuyNothingDay today, as a way to think about my impact on the planet and in society as a consumer".

Inequality. The category of inequality included tweets that referenced the growing income gap and class division in society. Mentions of consumption leading to the "rich getting richer," and other societal ill-effects were counted in this category. An example of a tweet revealing this motivation is: "The Rich Get Richer and The Poor Get Poorer Only IF We Remain Apathetic! Stop Being Mindless \#Consumers! \#BuyNothingDay Not \#BlackFriday”.

Anti-capitalism. Tweets referencing any form of corporate domination, big business, greed, excessive profits or Black Friday as a scam created by marketers to sell more stuff were coded as exhibiting an anti-capitalism motivation. In addition, tweets disparaging or criticizing a specific brand (Walmart and Target) were included in this category. For example, the following tweets were classified as showcasing an anti- capitalism motivation: "On this day let's encourage each other not to buy things on this repulsive capitalist holiday. \#BlackFriday \#buynothingday," and "I'm counting my blessings on \#buynothingday! No need to feed the capitalist monster".

Financial responsibility. Tweets in the category of financial responsibility referenced saving big by not spending at all, avoiding the regret of overspending and reminders to not spend money that you do not have. The following tweets sum up this sentiment: "Pay ya bills first! \#buynothingday" and: "\#BuyNothingDay \#BlackFriday \#spending If you have to charge it, you don't have it. Every year people go into debt trying to please other people buying things that they can't afford. Love is free. Make wise choices."

Financial necessity. A separate category of financial motivations was needed to capture tweets that indicated consumers could not participate in BND for financial reasons. Mentions that every day was BND because they were broke, or they could not afford to buy anything on Black Friday fell under this category. For example, the following tweet illustrates the financial necessity motivation: "It's so easy to celebrate \#BuyNothingDay when it coincides with HaveNothingToSpendDay".

Health. Tweets in the category of health revealed that participation in BND was for personal health, such as avoiding stress, fatigue, general unhappiness or physical injury because of aggressive shoppers. For example, the tweets "\#BlackFriday really stresses me 
out. I'm opting for \#BuyNothingDay!" and "A much healthier alternative to \#BlackFriday [...] \#BuyNothingDay" both exemplify this motivation. In addition, the following tweet reveals a motivation regarding physical health: "It's not worth fighting the crowds. And I may get trampled to death \#buynothingday".

Ethics. The category of ethics included tweets that brought up themes about the well-being of those retail employees who were working on BND, ethical divesting and charitable giving, and the benefits of buying local. An example of a tweet revealing this motivation is: "It should be illegal for \# retail stores to make their employees work on Thanksgiving and other holidays! Only doctors and that kind of necessary thing. \#BuyNothingDay \#BlackFriday”. Other examples which highlight BND as an opportunity to engage in ethical giving include: "Use your \#BuyNothingDay to \#GiveAway and help others today. \#Halifax \# extremecold" and “\#BuyNothingDay AND give something away".

Resistance to American culture. Some tweets identified Black Friday as a part of American culture and indicated that this American tradition had no place in other parts of the world. These tweets thus revealed a motivation to participate in BND to resist the "Americanisation" of their own culture. For instance, the tweets "\#BuyNothingDay A day for avoiding the shops and ignoring the dreadful consumerist US import of "black Friday" and "\#BuyNothingDay - Send Black Friday back to the stupid Americans where it belongs" reveal that some consumers participate in BND as a form of resistance toward what they perceive to be an unappealing American tradition.

No-motivation: positive. Tweets regarding BND that did not express a consumer's specific motivation for participation, but which did reveal consumer support for the event, were classified as no-motivation positive. For example, the tweet "Happy \#BuyNothingDay" does not imply a specific motivation for participation but indicate support or a positive impression of BND. Similarly, the following tweets exemplify the nomotivation positive category: "I didn't know it was a thing but I guess I'm in! \#BuyNothingDay" and "I'm so excited to buy absolutely nothing today \#BuyNothingDay”.

No-motivation: negative. Tweets that revealed no motivation for participation in and a negative sentiment toward BND were classified as no-motivation negative. For example, "Don't you tell me when to shop or if I needed or not \#buynothingday" was classified as no-motivation negative. Similarly, the following tweet reveals a negative sentiment 
toward BND and was coded as such: "\#BuyNothingDay Up yours \#BuyswhatIwantwhenIwant".

No-motivation: neutral. The no-motivation neutral code was used for tweets that satisfied two criteria: first, the tweet did not express a specific motivation for participating in BND, and second, the sentiment toward the event, as expressed in the tweet, was indiscernible. Most of the nomotivation neutral tweets were short tweets that simply mentioned BND without expressing a sentiment toward the event or indicating whether the individual was participating in it or not. For example, "Remember. \#BlackFriday \#BuyNothingDay," "Are you celebrating \#BlackFriday or \#BuyNothingDay?" or simply "\#BuyNothingDay" were tweets that meet these two criteria and were thus coded as "no- motivation neutral" tweets.

\section{Automated content analysis}

The automated content analysis used analysis techniques from the extant literature. The AI application Watson was deemed appropriate as it had been used in other studies to extract insights from user-generated data (Pitt et al., 2018a, 2018b). Moreover, the Watson application is particularly well suited to analyze Twitter data (Biondi et al., 2017) as its algorithms were trained on a large data sets of text data from social networking sites. The latter is important as the language in usergenerated content on social networking sites often lacks proper structure and uses informal language (Cvijikj and Michahelles, 2011; Yassine and Haij, 2010) which adds to the complexity in using automated content analysis techniques. Watson is therefore well suited to analyze large data sets (Cvijikj and Michahelles, 2011; Yassine and Haij, 2010). In the present study, the entire Twitter data set was subjected to content analysis, instead of only a sample of each year's data set.

Watson can process large volumes of data in short periods of time, making it a sophisticated and efficient method for content analysis of unstructured data, such as text (Balducci and Marinova, 2018; Duan et al., 2019; Gupta et al., 2018; Syam and Sharma, 2018). Two key components of the Watson application are its "natural language understanding" and "machine learning". The latter encompasses computational methods that enable AI systems to "learn from experience"; that is, to progressively improve their performance with 
increasing amounts of data and analysis (Paschen et al., 2019; Russell, 1997; Russell and Norvig, 2016; Tecuci, 2012). Natural language understanding, on the other hand, involves assigning meaning to text, (i.e. creating a semantic representation of the text) (High, 2012; Paschen et al., 2019; Zhu et al., 2014). Inherent to assigning meaning involves AI systems analyzing the syntax (i.e. the structure of sentences), semantics (i.e. the relationships between words, phrases and symbols) and pragmatics, (i.e. the context in which the words or phrases are used) (Gill, 2017).

In addition to extracting semantic themes, keywords or entities from text, Watson can provide insights into the human values. This is accomplished by extracting the four value orientations explained earlier based on Schwartz's (2012) value model: self-enhancement, selftranscendence, openness to change and conservation. Watson returns the scores for each value orientation as percentiles ranging from 0 to 1 ; a score above 0.5 indicate a greater than average tendency for a specific value orientation to be portrayed in the text while scores at 0.75 or above indicate readily discernible aspects of a specific value orientation (IBM, 2018).

\section{Results}

\section{Manual coding results}

Of the final data set, 2,188 tweets revealed at least one motivation for participation in BND. The frequency distribution (per cent) for the motivations is presented in Table II. A total of 3,347 tweets did not reveal a motivation and were classified based on valence as no- motivation positive, negative or neutral (Table III). 
Table II. Manual coding results of motivation for participation in BND (2016-2018)

\begin{tabular}{|lr|}
\hline Frequency of motivation & (\%) \\
\hline Personal welfare & 30 \\
Consumerism & 26 \\
Wastefulness & 15 \\
Environmental concerns & 13 \\
Anti-capitalism & 8 \\
Financial responsibility & 6 \\
Ethics & 6 \\
Financial necessity & 5 \\
Inequality & 3 \\
Health & 2 \\
Resistance to American culture & 2 \\
\hline
\end{tabular}

Table III. Manual coding results of attitude toward BND in tweets without motivations (2016-2018)

\begin{tabular}{|lr|}
\hline Valence toward BND & (\%) \\
\hline Personal welfare & 65 \\
Consumerism & 28 \\
Wastefulness & 7 \\
\hline
\end{tabular}

\section{Artificial intelligence results}

The Twitter data was input into the AI application Watson to derive the four value dimensions using Schwartz' $(1992,2012)$ value model (Table IV). The results suggest that the value dimensions "conservation" and "self- enhancement" have a lower than average representation in the BND tweets (percentile scores of 0.23 and 0.30 , respectively), while the value dimensions "self-transcendence" and "openness to change" are readily discernible in the BND tweets (percentile scores of 0.51 and 0.62 ), respectively.

Table IV. Al results of value dimensions using Schwartz' $(1992,2012)$ value model in BND tweets (2016-2018)

\begin{tabular}{|lr|}
\hline Value dimension & Percentile \\
\hline Conservation & 0.23 \\
Openness to change & 0.62 \\
Self-enhancement & 0.30 \\
Self-transcendence & 0.51 \\
\hline
\end{tabular}




\section{Discussion and contributions}

\section{Contributions to the literature on consumption restraint}

Our research investigated the motivations and values toward consumption restraint expressed in tweets about a major consumption restraint event. Overall, our manual analysis revealed that most consumers who tweeted about BND had a positive attitude toward it, and nearly all of the tweets that revealed the consumer's motivation were in support of BND, and the majority of tweets without clear motivations were also inferred as positive.

The motivations identified from the manual content analysis are supported by findings from previous research. Specifically, consumerism and personal welfare were by far the most common motivators identified in our analysis. This finding is well aligned with current research indicating that excessive consumption, as in the context of Black Friday, may have negative psychological impacts (Zavestoski, 2002). In the face of these negative impacts, many consumers tweeted about spending their time engaging in an enjoyable personal activity instead of shopping. In addition, while our analysis is specific to BND, the prevalence of these two factors may reveal overall trends in consumer behavior. That is, it is possible that these motivations are reflective of larger trends toward a desire to consume less and experience more or simply to be more mindful when making purchase decisions (Sheth et al., 2011). Indeed, mindful consumption (Sheth et al., 2011) involves both the consumer mindset and their behavior (e.g. consumption temperance) and is characterized by a sense of caring for the self, community and nature. These three aspects of mindful consumption are well aligned with the motivations found for participation in BND. As such, our results resonate with those of Sheth et al. (2011) in which mindful consumption is conceptualized as a central point of sustainable marketing. Other research suggests that millennials may lead a larger trend away from consumers' focusing on consumption toward a focus on well-being (Brown and Vergragt, 2016) which also aligns with our results.

The finding that a negative attitude toward consumerism was consistently a top motivator for participation in BND expressed via Twitter is not surprising, as BND originated from the anti-consumerist organization Adbusters and many of Adbusters' messages about the event 
carry warnings about over-consumption in today's society. Thus, the prominent sentiment regarding consumerism may reflect effective positioning of BND by its creators. Beyond this, many consumers, regardless of their affinity to participate in a consumption temperance movement or of their awareness of the origin of the BND movement, are likely aware of Black Friday's reputation for being a frenzied shopping environment and find it unappealing. As the hype and scale of Black Friday intensifies, some consumers are likely to respond by opting to avoid this experience all together.

The theme related to physical and psychological health is also well aligned with existing research which indicates that excessive consumption can lead to feelings of stress, disillusionment, unhappiness or fatigue (Zavestoski, 2002). Furthermore, in recent years, Black Friday shopping events have received negative media attention because of images of shoppers trampling over each other and crashing through doors at midnight to access the hottest deals. In some cases, Black Friday sales have been linked to consumer deaths because of trampling or other violence, and there is a website dedicated to tallying yearly fatalities linked to Black Friday (blackfridaydeathcount.com). The finding that participants of BND referenced their physical safety concerns is indeed justified, as research that shows scarcity promotions such as those common in Black Friday sales increase aggression among shoppers (Kristofferson et al., 2016). As these unintended consequences from scarcity promotions receive more and more press coverage, consumers may increasingly bring these up as motivators for avoiding Black Friday.

Outside of the specific event of Black Friday, BND is also intended to give consumers pause to reflect broadly on their consumption behaviors throughout the year. It is no surprise then that wastefulness and the environmental impact of consumption were cited by Twitter users supporting BND. For many green consumers, non-consumption is a critical component of sustainability, as it is a form of conservation (Black and Cherrier, 2010). Given that consumption restraint is known to stem from an individual's values and beliefs, tweeting a message about the environment on BND could be a way of expressing one's self- identity. Indeed, because environmental concerns are known to be important to consumers, one surprising finding was that environmentalism was not a more prevalent motivating factor in consumer tweets regarding participation in BND. The fact that more 
tweets did not reveal this motivation suggests that a typical consumer who does not consider him or herself to be an activist, may be less concerned with or aware of the environmental implications of their consumption choices.

Financial necessity and financial responsibility are two motivations found in this research that have not been revealed in the current literature regarding consumer activists or anti-consumers, but rather only in research focusing on non-voluntary restraint (Beninger and Robson, 2015; Leipämaa-Leskinen et al., 2016). Thus, our research reveals that to a typical consumer, restraint can be associated with their financial position - either the consumer restrains from consumption because they do not have the disposable income or because they view restraint as the most responsible financial decision. These motivations set typical consumers apart from consumer activists.

The AI analysis of human values also provides a number of contributions to the literature on consumption restraint. The results show that the value dimension "conservation" has a lower than average representation in the BND tweets. This finding is supported by previous research with self-identified socially conscious consumers (Pepper et al., 2009). The conservation dimension relates to the values of security (i.e. the desire for harmony and stability of one's relationships with others and with self), conformity (i.e. self- restraint in everyday interactions with others) and tradition (i.e. respect and acceptance of the customs and ideas provided by society) (Schwartz, 2012). As the majority of tweets were in support of BND, one would expect participants to have a lower than average representation on values that emphasize tradition, conformity and security. As revealed in the motivations from our manual analysis, a negative attitude toward the common societal practices of consumerism was among the most frequently discerned motivations in the tweets.

The results from the AI analysis also reveal a lower than average score on the value dimension "self-enhancement," which includes values related to achievement (i.e. personal success), power (i.e. social status, prestige, dominance over others or over resources) and hedonism (i.e. gratification for oneself). This finding aligns with other research with selfidentified consumers practicing consumption restraint (Pepper et al., 2009). This finding is not surprising, as the BND movement calls into question existing dominance efforts by firms or consumer dominance 
over limited resources, as expressed in the motivations related to consumerism, environmental or ethical concerns.

With respect to the remaining two value dimensions, the findings suggest that "self- transcendence" and "openness-to-change" are represented more frequently than average in the BND tweets, albeit only slightly for self-transcendence. "Self-transcendence" consists of universalism (i.e. concern for the welfare of all people and for nature) and benevolence (i.e. preserving and enhancing the welfare of close relationships) while "openness to change" entails self-direction (i.e. independent thought and action) and stimulation (i.e. novelty and challenge in life). With respect to self-transcendence, the result may be surprising at first glance as consumption restraint behavior is often motivated by social concerns, such as concerns about the environment or material inequality (Iyer and Muncy, 2009). Thus, one may expect a higher score for the self-transcendence value-dimension. One possible explanation for the only slightly higher score in our study is that "benevolence" (a value type included in self-transcendence) requires positive interaction and affiliation with close relationships (Shaw et al., 2005) which may not be intended by BND participants. On the contrary, the prevalence of a negative attitude toward consumerism as one of the main motivations expressed in BND tweets may be an indication for consumers trying to distance themselves from the consumption frenzy of other consumers, including their close relationships. Lastly, the higher than average score on "openness to change" is well aligned with the existing literature on consumption restraint where independent thought and action and challenge in life may be an expression of resistance against power forces exhibited by firms or other consumers.

\section{Contributions to the literature on content analysis}

This interpretation of the data also reiterates the utility of combining manual and automated content analysis techniques into a hybrid method. Specifically, the research in this paper suggests that machine capabilities can be enhanced by humans, specifically when it comes to interpreting the results of automated content analysis. While automated tools, such as AI applications, extract information from large volumes of text data, human skills are needed to turn this information into knowledge, such as 
the implications for managerial decision- making. In this regard, AI can do the "heavy lifting" by processing large data sets, for instance via its natural language understanding and its machine-learning components. Thus, the value that AI can bring is primarily of analytical nature (i.e. data analysis and processing). The value-add contributions from humans lie primarily in interpreting the AI- generated information and deriving insights and implications for marketing decision- making. In addition, humans explaining the automated results are critical, particularly when the results are counter-intuitive or even contradictory, as is the case in our discussion of the seemingly contrary findings regarding the "selftranscendence" value dimension expressed in tweets.

Thus, AI as one specific automated content analysis application can both complement and augment human capabilities (Jarrahi, 2018), an approach that has recently been termed "collaborative intelligence" (Wilson and Daugherty, 2018). Indeed, the use of AI may be particularly useful when it comes to extracting information about consumers' underlying psychological attributes or processes, such as values from large volumes of data. AI algorithms, with their ability to analyze large volumes of text, can derive information more efficiently than humans would. In addition, by analyzing content voluntarily created by consumers themselves, marketers may obtain insight that otherwise would be difficult to gain with more traditional consumer research approaches, such as interviews or surveys, where consumers may be reluctant or incapable to offer the required information. Thus, AI has the potential to enhance the effectiveness and efficiency of human approaches to deriving consumer insights (Jarrahi, 2018; Paschen et al., 2020). Lastly, our research provides a methodological contribution by demonstrating a robust and impactful method of understanding human values whereby the limitations of response bias, lacking self- awareness, socially desired responses and the time and effort required from consumers completing a value survey can be minimized.

\section{Implications for practice}

For marketing managers, our study provides a more nuanced understanding of the motivations and values that a broad population has toward consumption restraint. Mining user-generated content and listening in, particularly on social media, is known to be an effective 
approach for marketing communications managers who wish to understand consumers' responses to their messages (Balducci and Marinova, 2018). Thus, managers may derive value from our insights both for their long-term as well as short-term communication strategies and tactics. In the short term, marketing managers, particularly in retail, may use the insights derived from our study to inform their communication tactics for holiday advertising and promotions. For example, it is possible that given the overall positive sentiment toward $\mathrm{BND}$, choosing to close shop on BND may actually serve as a differentiating factor for an organization. Several retailers already do this; REI, for example, has closed their doors on Black Friday since 2015, and promoted an anti-Black Friday campaign called \#OptOutside in which they encouraged consumers to do what they loved in the outdoors (Coffee, 2016). In the past, Patagonia has also chosen to close their doors and in years when they have remained open, they have often donated 100 per cent of their sales to non-profit environmental organizations (Monllos, 2016). These business actions could be considered forms of conscious capitalism (O'Toole and Vogel, 2011) and may be appealing to many consumers who are conflicted about participating in Black Friday consumption.

In the long term, marketers can use the findings from this research to consider themes to embed into their future promotional campaigns. In addition, managers are well-advised to monitor user-generated communication on social media as these communications can influence buyer and customer preferences and behaviors (Berger $e t$ al., 2019; Frow and Payne, 2011; Kohli and Jaworski, 1990). This information is valuable for marketing managers as it can inform their longer-term marketing communication strategies, such as to how they position their brand or offerings. Moreover, our analysis derived 11 distinct motivations for participating in consumption restraint, which managers can use as a basis for market segmentation or to inform their targeting and positioning strategies. Finally, the hybrid content analysis approach used in this paper can serve as a template for managers and future scholarly research alike, particularly when it comes to analyzing unstructured text data from social media communications. AI, with its natural language understanding and machine-learning elements, is particularly well-suited to analyze text data that may use conversational language or be incorrectly spelled. 
This provides managers with the opportunities to gain valuable knowledge about their market effectively and efficiently.

\section{Limitations and future research}

Although the BND movement has gained popularity, much is left to be understood about how the typical consumer exercises restraint in their consumption. We discuss some of these avenues for further research below, as well as some of the limitations of our work. Future research would benefit from exploration of the managerial approaches to dealing with consumers who practice consumption restraint. That is, examination of the business or retailer response to either temperance on Black Friday or more specifically the BND movement, would be insightful. Investigating how consumers perceive brands that encourage some form of consumption restraint could be informative.

Consumer reactions to such actions, as well as their links to the personal and environmental motivations uncovered in this research, are aspects that we encourage researchers to investigate in the future. For example, future research could explore the themes identified in tweets and whether these themes are linked with the likelihood of retweets, likes or other reactions. In addition, future research could explore whether the personality information of each user (i.e. the information provided in Twitter profiles) sheds additional light on consumer participation in BND.

Future research would also benefit from work that adds depth to the current study. For example, future qualitative research could explore the typical consumer's motivations for acts of consumer resistance through in-depth qualitative exploration such as interviews. More detailed conversations with consumers could help to answer important questions, including: Are events such as BND effective at restraining an individual's consumption or do they merely shift the time-period in which they consume? Do consumers share their participation in BND because of what it says about them?

Like all research studies, this work has a number of limitations that must be acknowledged. Our inclusion criteria were designed to identify and include all tweets regarding BND; however, there are a number of reasons why our tweet sample is unlikely to be comprehensive. Specifically, some consumers are likely to have restrained from Black 
Friday and not tweeted about it, and others likely participated and tweeted about this restraint, but without the use of the BND term or hashtag. In addition, it is possible that a number of consumers either misspelled the term or hashtag which would have resulted in the tweet being excluded from our data set. Because of language restrictions of the researchers, all tweets included in the analysis in this work were written in English. However, BND occurs in 65 countries around the world, many of which do not have English as their first language. As a result, it is possible that the sample of tweets analyzed in this work reflects a particular subset of consumers who tweeted about BND in English, which might differ from consumers who tweeted in other languages. Future research could assess cultural differences in consumer motivations for participation in BND or other consumption restraint events. Finally, although our goal was to understand motivations of typical consumers, it is possible that some of those who tweeted about their participation in BND were individuals who selfidentify as culture jammers, voluntary simplifiers or other types of activists.

\section{Conclusion}

This article investigated the motivations and values that a general sample of consumers has to participate in consumption restraint behavior. We explored this topic by conducting a hybrid content analysis of tweets referencing BND during the 2016, 2017 and 2018 Black Friday shopping seasons. Several themes emerged from the content analysis to suggest that consumers have multiple reasons for engaging in this practice. In addition, the content analysis suggested that specific human values are prominent among BND participants. Our study offers contributions to both the academic literature and to practice. For academics, to the best of our knowledge, this is the first study investigating the consumption restraint motivations of "typical" consumers on a broad scale. Previous studies have primarily focused on self-identified consumer resistance groups with small samples. Our investigation analyzes the motivations to participate in consumption restraint using a large, public data set. We thereby add to a more robust understanding of consumption restraint 
behavior and our work identifies two new motivations previously not discussed in the literature. In addition, this research adds to the body of literature that suggests hybrid methods of content analysis are an effective approach to extracting valid and reliable insights from textbased data. Our study also has implications for practice. Our findings provide insights into why the general public may engage in consumption restraint behavior which can be valuable for organizational decisionmaking, for example to craft appropriate strategies in response to consumption restraint. 


\section{References}

Albinsson, P.A., Wolf, M. and Kopf, D.A. (2010), "Anti-consumption in East Germany: consumer resistance to hyperconsumption", Journal of Consumer Behaviour, Vol. 9 No. 6, pp. 412-425.

Asur, S. and Huberman, B.A. (2010), "Predicting the future with social media", Proceedings of the $2010 \mathrm{IEEE} / \mathrm{WIC} / \mathrm{ACM}$ International Conference on Web Intelligence and Intelligent Agent Technology- Volume 01, IEEE Computer Society, pp. 492-499.

Avery, E., Lariscy, R. and Sweetser, K.D. (2010), "Social media and shared - or divergent - uses? A coorientation analysis of public relations practitioners and journalists", International Journal of Strategic Communication, Vol. 4 No. 3, pp. 189-205.

Balderjahn, I., Peyer, M., Seegebarth, B., Wiedmann, K.P. and Weber, A. (2018), "The many faces of sustainability-conscious consumers: a category-independent typology", Journal of Business Research, Vol. 91, pp. 83-93.

Balducci, B. and Marinova, D. (2018), "Unstructured data in marketing", Journal of the Academy of Marketing Science, Vol. 46 No. 4, pp. 557-590.

Beninger, S. and Robson, K. (2015), "Marketing at the base of the pyramid: perspectives for practitioners and academics", Business Horizons, Vol. 58 No. 5, pp. 509-516.

Berger, J., Humphreys, A., Ludwig, S., Moe, W.W., Netzer, O. and Schweidel, D.A. (2019), "Uniting the tribes: using text for marketing insight", Journal of Marketing.

Biondi, G., Valentina F., and Valentina P. (2017). "A deep learning semantic approach to emotion recognition using the IBM Watson bluemix alchemy language." In International Conference on Computational Science and Its Applications, pp. 718-729. Springer, Cham.

Black Friday's travels around the world (2015), "BBC news, 27 November", available at: www.bbc.com/news/av/business-34939238/black-friday-s-travels-aroundthe-world (accessed 27 December 2018).

Black, I.R. and Cherrier, H. (2010), "Anti-consumption as part of living a sustainable lifestyle: daily practices, contextual motivations and subjective values", Journal of Consumer Behaviour, Vol. 9 No. 6, pp. 437-453.

Britannica Academic (2020), "Buy nothing day", available at: http://academic.eb.com.cmich.idm.oclc. org/levels/collegiate/article/Buy-NothingDay/605851 (accessed 25 March 2017).

Brown, H. Guskin, E. and Mitchell, A. (2012), "The role of social media in the Arab uprisings, pew research center", available at: www.journalism.org/2012/11/28/rolesocial-media-arab-uprisings/

Brown, H.S. and Vergragt, P.J. (2016), "From consumerism to wellbeing: toward a cultural transition?", Journal of Cleaner Production, Vol. 132, pp. 308-317.

Burch, L.M., Frederick, E.L. and Pegoraro, A. (2015), "Kissing in the carnage: an examination of framing on twitter during the Vancouver riots", Journal of Broadcasting and Electronic Media, Vol. 59 No. 3, pp. 399-415.

Cheng, A.S., Fleischmann, K.R., Wang, P., Ishita, E. and Oard, D.W. (2012), "The role of innovation and wealth in the net neutrality debate: a content analysis of human values in congressional and FCC hearings", Journal of the American Society for Information Science and Technology, Vol. 63 No. 7, pp. 1360-1373.

Cherrier, H. (2009), "Anti-consumption discourses and consumer-resistant identities", Journal of Business Research, Vol. 62 No. 2, pp. 181-190. 
Cherrier, H. and Murray, J.B. (2007), "Reflexive dispossession and the self: constructing a processual theory of identity", Consumption Markets and Culture, Vol. 10 No. 1, pp. 1-29.

Cherrier, H., Black, I.R. and Lee, M. (2011), "Intentional non-consumption for sustainability: consumer resistance and/or anti-consumption?”, European Journal of Marketing, Vol. 45 Nos 11/12, pp. 1757-1767.

Coffee, P. (2016), "REl's anti-black friday campaign \#OptOutside wins the titanium grand prix at Cannes", available at: www.adweek.com/brand-marketing/reis-anti-blackfriday-campaign- optoutside-wins-titanium-grand-prix-cannes-172256/ (accessed 25 March 2017).

Conway, M. (2006), "The subjective precision of computers: a methodological comparison with human coding in content analysis", Journalism and Mass Communication Quarterly, Vol. 83 No. 1, pp. 186-200.

Craig-Lees, M. and Hill, C. (2002), "Understanding voluntary simplifiers", Psychology and Marketing, Vol. 19 No. 2, pp. 187-210.

Cronin, J.M., McCarty, M. and Delaney, M. (2015), "Deconstructing consumer discipline: how self- management is experienced in the marketplace", European Journal of Marketing, Vol. 49 Nos 11/12, pp. 1902-1922.

Cvijikj, I.P. and Michahelles, F. (2011), "Understanding social media marketing: a case study on topics, categories and sentiment on a Facebook brand page", Proceedings of the 15th International Academic MindTrek Conference: Envisioning Future Media Environments, ACM, pp. 175-182.

Da, Z., Engelberg, J. and Gao, P. (2011), "In search of attention", The Journal of Finance, Vol. 66 No. 5, pp. 1461-1499.

Dabirian, A., Kietzmann, J.H. and Diba, H. (2017), "A great place to work!? Understanding crowdsourced employer branding", Business Horizons, Vol. 60 No. 2, pp. 197-205.

Dobscha, S. (1998), "The lived experience of consumer rebellion against marketing", NA-Advances in Consumer Research Volume 25, available at: www.acrwebsite.org/volumes/8131/volumes/v25/ NA-25 (accessed 25 March 2017).

Domo (2018), "Data never sleeps 6.0: how much data is generated every minute", Domo Learn Center, available at: www.domo.com/learn/data-never-sleeps-6

Duan, Y., Edwards, J.S. and Dwivedi, Y.K. (2019), "Artificial intelligence for decision making in the era of big data-evolution, challenges and research agenda", International Journal of Information Management, Vol. 48 No. 48, pp. 63-71.

Eagle, L. and Dahl, S. (2015), Marketing Ethics and Society, Sage, available at: https://books.google.com/ books?hl=en\&l $\mathrm{r}=\& \mathrm{id}=9$ COJCwAAQBAJ\&oi=fnd\&pg=PP1\&dq=EaglepDahlp2015\&ots= fmK3fWYFHS\&sig=cQYeikeZpsvi2U8HdOzJp9T43H8 (accessed 25 March 2017).

Evans, D. (2011), "Thrifty, green or frugal: reflections on sustainable consumption in a changing economic climate", Geoforum, Vol. 42 No. 5, pp. 550-557.

Frow, P. and Payne, A. (2011), "A stakeholder perspective of the value proposition concept", European Journal of Marketing, Vol. 45 Nos 1/2, pp. 223-240.

Füller, J. (2010), "Refining virtual co-creation from a consumer perspective", California Management Review, Vol. 52 No. 2, pp. 98-122.

Galvagno, M. (2011), "The intellectual structure of the anti-consumption and consumer resistance field: an author co-citation analysis", European Journal of Marketing, Vol. 45 Nos 11/12, pp. 1688-1701.

Garcia-Bardidia, R., Nau, J.-P. and Rémy, E. (2011), "Consumer resistance and anticonsumption: insights from the deviant careers of French illegal downloaders", European Journal of Marketing, Vol. 45 Nos 11/12, pp. 1789-1798. 
Gilg, A., Barr, S. and Ford, N. (2005), "Green consumption or sustainable lifestyles? Identifying the sustainable consumer", Futures, Vol. 37 No. 6, pp. 481-504.

Gill, N.S. (2017), "Overview of artificial intelligence and natural language processing", available at: www.upwork.com/hiring/for-clients/artificial-intelligence-andnatural-language-processing-in-big- data/ (accessed 11 December 2017).

Goel, S., Hofman, J.M., Lahaie, S., Pennock, D.M. and Watts, D.J. (2010), "Predicting consumer behavior with web search", Proceedings of the National Academy of Sciences, Vol. 107 No. 41, pp. 17486-17490.

Grimmer, J. and Stewart, B.M. (2013), "Text as data: the promise and pitfalls of automatic content analysis methods for political texts", Political Analysis, Vol. 21 No. 3, pp. 267-297.

Gruhl, D., Guha, R., Kumar, R., Novak, J. and Tomkins, A. (2005), "The predictive power of online chatter", Proceedings of the Eleventh ACM SIGKDD International Conference on Knowledge Discovery in Data Mining, ACM, New York, NY, pp. 78-87.

Gupta, S., Kar, A.K., Baabdullah, A. and Al-Khowaiter, W.A. (2018), "Big data with cognitive computing: a review for the future", International Journal of Information Management, Vol. 42, pp. 78-89.

High, R. (2012), The Era of Cognitive Systems: An inside Look at IBM Watson and How It Works, IBM Corporation, retrieved from http://johncreid.com/wpcontent/uploads/2014/12/The-Era-of-Cognitive-Systems-An-Inside-Look-at-IBMWatson-and-How-it-Works_.pdf.

Hoffmann, S. (2011), "Anti-consumption as a means to save jobs", European Journal of Marketing, Vol. 45 Nos 11/12, pp. 1702-1714.

Hogg, M.K., Banister, E.N. and Stephenson, C.A. (2009), "Mapping symbolic (anti-) consumption", Journal of Business Research, Vol. 62 No. 2, pp. 148-159.

Humphreys, A. and Wang, R.J.-H. (2017), "Automated text analysis for consumer research", Journal of Consumer Research, Vol. 44 No. 6, pp. 1274-1306.

Humphreys, L., Gill, P. and Krishnamurthy, B. (2014), "Twitter: a content analysis of personal information", Information, Communication and Society, Vol. 17 No. 7, pp. 843857.

IBM (2018), "Personality insights", IBM, 21 August, available at: https://console.bluemix.net/docs/services/personalityinsights/conscientiousness.html\#conscientiousness

Iyer, R. and Muncy, J.A. (2009), "Purpose and object of anti-consumption", Journal of Business Research, Vol. 62 No. 2, pp. 160-168.

Jägel, T., Keeling, K., Reppel, A. and Gruber, T. (2012), "Individual values and motivational complexities in ethical clothing consumption: a means-end approach", Journal of Marketing Management, Vol. 28 Nos 3/4, pp. 373-396.

James, P. and Szeman, I. (2010), "Globalization and culture, vol. 3: global-local consumption", available at:

www.academia.edu/4501557/Globalization_and_Culture_Vol._3_Global-

Local_Consumption_2010 (accessed 25 March 2017).

Jarrahi, M.H. (2018), "Artificial intelligence and the future of work: human-Al symbiosis in organizational decision making", Business Horizons, Vol. 61 No. 4, pp. 577-586.

Jin, S.A.A. and Pua, J. (2014), "Following celebrities' tweets about brands: the impact of twitter-based electronic word-of-mouth on consumers' source credibility perception, buying intention, and social identification with celebrities", Journal of Advertising, Vol. 43 No. 2, pp. 181-195. 
Kaplan, A. and Haenlein, M. (2019), "Siri, Siri, in my hand: who's the fairest in the land? On the interpretations, illustrations, and implications of artificial intelligence", Business Horizons, Vol. 62 No. 1, pp. 15-25.

Karpf, D. (2012), "Social science research methods in internet time", Information, Communication and Society, Vol. 15 No. 5, pp. 639-661.

Kassarjian, H.H. (1977), "Content analysis in consumer research", Journal of Consumer Research, Vol. 4 No. 1, pp. 8-18.

Kietzmann, J.H., Hermkens, K., McCarthy, I.P. and Silvestre, B.S. (2011), "Social media? Get serious! Understanding the functional building blocks of social media", Business Horizons, Vol. 54 No. 3, pp. 241-251.

Kohli, A.K. and Jaworski, B.J. (1990), "Market orientation: the construct, research propositions, and managerial implications", Journal of Marketing, Vol. 54 No. 2, pp. 1-18.

Kozinets, R.V. and Handelman, J.M. (2004), "Adversaries of consumption: consumer movements, activism, and ideology", Journal of Consumer Research, Vol. 31 No. 3, pp. 691-704.

Kristofferson, K., McFerran, B., Morales, A.C. and Dahl, D. (2016), "The dark side of scarcity promotions: how exposure to limited-quantity promotions can induce aggression", Journal of Consumer Research, Vol. 43 No. 5, pp. 683-706.

Lacy, S., Watson, B.R., Riffe, D. and Lovejoy, J. (2015), "Issues and best practices in content analysis", Journalism and Mass Communication Quarterly, Vol. 92 No. 4, pp. 791811.

Lai, L.S. and To, W.M. (2015), "Content analysis of social media: a grounded theory approach", Journal of Electronic Commerce Research, Vol. 16 No. 2, p. 138.

Lee, M., Roux, F., Cherrier, G. and Cova, B. (2011), "Anti-consumption and consumer resistance: concepts, concerns, conflicts and convergence", European Journal of Marketing, Vol. 45 Nos 11/12.

Lee, M.S.W., Fernandez, K. and Hyman, M.R. (2009), "Anti-consumption: an overview and research agenda", Journal of Business Research, Vol. 62 No. 2, pp. 145-147.

Leipämaa-Leskinen, H., Syrjälä, H. and Laaksonen, P. (2016), "Conceptualizing nonvoluntary anti- consumption: a practice-based study on market resistance in poor circumstances", Journal of Consumer Culture, Vol. 16 No. 1, pp. 255-278.

Lewis, S.C., Zamith, R. and Hermida, A. (2013), "Content analysis in an era of big data: a hybrid approach to computational and manual methods", Journal of Broadcasting and Electronic Media, Vol. 57 No. 1, pp. 34-52.

Locke, K. and Golden-Biddle, K. (1997), "Constructing opportunities for contribution: structuring intertextual coherence and 'problematizing' in organizational studies", Academy of Management Journal, Vol. 40 No. 5, pp. 1023-1062.

Marr, B. (2018), "How much data do we create every day? The mind-blowing stats everyone should read", Forbes, 21 May, available at: www.forbes.com/sites/bernardmarr/2018/05/21/how-much- data-do-we-create-every-daythe-mind-blowing-stats-everyone-should-read/\#38ec8fd660ba

Monllos, K. (2016), "Patagonia will donate $100 \%$ of its Black Friday sales to helping the environment", 21 November, available at: www.adweek.com/brand-marketing/patagoniawill-donate-100-its- black-friday-sales-helping-environment-174751/ (accessed 25 March 2017).

Moore, R.J. (2009), “Twitter data analysis: an investor's perspective", TechCrunch, 5 October, available at: www.washingtonpost.com/wp-

dyn/content/article/2009/10/05/AR2009100504023.html 
Odou, P. and De Pechpeyrou, P. (2011), "Consumer cynicism: from resistance to anti-consumption in a disenchanted world?", European Journal of Marketing, Vol. 45 Nos 11/12, pp. 1799-1808.

OECD (2019), "Household debt (indicator)", available at: https://data.oecd.org/hha/household-debt.htm (accessed 30 April 2019).

O'Toole, J. and Vogel, D. (2011), "Two and a half cheers for conscious capitalism", California Management Review, Vol. 53 No. 3, pp. 60-76.

Paschen, J. (2019), "Investigating the emotional appeal of fake news versus real news using artificial intelligence", Journal of Product and Brand Management, doi: 10.1108/JPBM-12-2018-2179.

Paschen, J., Kietzmann, J. and Kietzmann, T. (2019), "Artificial intelligence (Al) and its implications for market knowledge in B2B marketing", Journal of Business and Industrial Marketing.

Paschen, J., Wilson, M. and Ferreira, J. (2020), "Collaborative intelligence - how human and artificial intelligence (AI) create value along the B2B sales funnel", Business Horizons,

Paschen, J., Pitt, L., Kietzmann, J., Dabirian, A. and Farshid, M. (2017), "The Brand personalities of brand communities: an analysis of online communication”, Online Information Review, Vol. 41 No. 7, pp. 1064-1075.

Penaloza, L. and Price, L.L. (1993), "Consumer resistance: a conceptual overview", NA-Advances in Consumer Research Volume 20, available at:

www.acrwebsite.org/search/view-conference- proceedings.aspx?ld=7423 (accessed 25 March 2017).

Pentina, I. and Amos, C. (2011), "The Freegan phenomenon: anti-consumption or consumer resistance?”, European Journal of Marketing, Vol. 45 Nos 11/12, pp. 17681778.

Pepper, M., Jackson, T. and Uzzell, D. (2009), "An examination of the values that motivate socially conscious and frugal consumer behaviours", International Journal of Consumer Studies, Vol. 33 No. 2, pp. 126-136.

Pitt, C., Kietzmann, J., Botha, E. and Wallström, Å. (2018a), "Emotions and sentiment: an exploration of artist websites", Journal of Public Affairs, Vol. 18 No. 2, p. 1653.

Pitt, C., Mulvey, M. and Kietzmann, J.H. (2018b), "Quantitative insights from online qualitative data: an example from the health care sector", Psychology and Marketing, Vol. 35 No. 12, pp. 1010-1017.

Pitt, C., Plangger, K.A., Botha, E., Kietzmann, J. and Pitt, L.F. (2017), "How employees engage with B2B brands on social media: word choice and verbal tone", Industrial Marketing Management, Vol. 81, pp. 130-137.

Qin, J. (2015), "Hero on twitter, traitor on news: how social media and legacy news frame Snowden", The International Journal of Press/Politics, Vol. 20 No. 2, pp. 166-184.

Raymond, M. (2010), "How tweet it is!: library acquires entire Twitter archive", Library of Congress Blog, Vol. 14.

Reynolds, T. and Smith, A. (2016), "137.4 Million Consumers Plan to Shop Thanksgiving Weekend", National Retail Federation, Text, 17 November, available at: https://nrf.com/media/press- releases/1374-million-consumers-plan-shop-thanksgivingweekend (accessed 27 March 2017).

Rindell, A., Strandvik, T. and Wilén, K. (2014), "Ethical consumers' brand avoidance", Journal of Product and Brand Management, Vol. 23 No. 2, pp. 114-120.

Robson, K., Kietzmann, J.H. and Pitt, L.F. (2016), "APC forum: extending business values through wearables”, MIS Quarterly Executive, Vol. 15 No. 2, pp. 169-179. 
Robson, K., Pitt, L. and West, D.C. (2015), "Navigating the peer-review process: reviewers' suggestions for a manuscript", Journal of Advertising Research, Vol. 55 No. 1, pp. 9-17.

Roux, D. (2007), "Consumer resistance: proposal for an integrative framework", Recherche et Applications en Marketing, Vol. 22 No. 4, pp. 59-79.

Russell, S.J. (1997), "Rationality and intelligence", Artificial Intelligence, Vol. 94 Nos 1/2, pp. 57-77. Russell, S.J. and Norvig, P. (2016), Artificial Intelligence: A Modern Approach, 3rd ed., Pearson Education, Upper Saddle River, NJ.

Schwartz, S.H. (1992), "Universals in the content and structure of values: theoretical advances and empirical tests in 20 countries", Advances in Experimental Social Psychology, Vol. 25, pp. 1-65.

Schwartz, S.H. (2012), "An overview of the Schwartz theory of basic values", Online Readings in Psychology and Culture, Vol. 2 No. 1, p. 11.

Schwartz, S.H. and Bilsky, W. (1987), "Toward a universal psychological structure of human values", Journal of Personality and Social Psychology, Vol. 53 No. 3, pp. 550-562.

Shah, R.C. and Kesan, J.P. (2003), "Manipulating the governance characteristics of code", Info, Vol. 5 No. 4, pp. 3-9.

Shaw, D., Grehan, E., Shiu, E., Hassan, L. and Thomson, J. (2005), "An exploration of values in ethical consumer decision making", Journal of Consumer Behaviour, Vol. 4 No. 3 , pp. $185-200$.

Sheth, J.N., Sethia, N.K. and Srinivas, S. (2011), "Mindful consumption: a customercentric approach to sustainability", Journal of the Academy of Marketing Science, Vol. 39 No. 1, pp. 21-39.

Short, J.C. and Palmer, T.B. (2003), "Organizational performance referents: an empirical examination of their content and influences", Organizational Behavior and Human Decision Processes, Vol. 90 No. 2, pp. 209-224.

Short, J.C. Broberg, J.C. Cogliser, C.C. and Brigham, K.C. (2009), "Construct validation using computer-aided text analysis (CATA): an illustration using entrepreneurial orientation", Organizational Research Methods, available at:

http://orm.sagepub.com/content/early/2009/05/05/1094428109335949.short (accessed 2 February 2017).

Short, J.C., McKenny, A.F. and Reid, S.W. (2018), "More than words? Computer-aided text analysis in organizational behavior and psychology research", Annual Review of Organizational Psychology and Organizational Behavior, Vol. 5 No. 1, pp. 415-435.

Singh, J., Flaherty, K., Sohi, R.S., Deeter-Schmelz, D., Habel, J., Le Meunier-FitzHugh, K., Malshe, A., Mullins, R. and Onyemah, V. (2019), "Sales profession and professionals in the age of digitization and artificial intelligence technologies: concepts, priorities, and questions", Journal of Personal Selling and Sales Management, Vol. 39, pp. 1-21.

Stueck, W. (2018), "A tale of two riots: the role of social media", Globe and Mail, 29 April, available at: www. theglobeandmail.com/news/british-columbia/a-tale-of-two-riots-therole-of-social-media/article583642/

Su, L.Y.-F., Cacciatore, M.A., Liang, X., Brossard, D., Scheufele, D.A. and Xenos, M.A. (2017), "Analyzing public sentiments online: combining human-and computer-based content analysis", Information, Communication and Society, Vol. 20 No. 3, pp. 406-427.

Syam, N. and Sharma, A. (2018), "Waiting for a sales renaissance in the fourth industrial revolution: machine learning and artificial intelligence in sales research and practice", Industrial Marketing Management, Vol. 69, pp. 135-146.

Tecuci, G. (2012), "Artificial intelligence", Wiley Interdisciplinary Reviews: Computational Statistics, Vol. 4 No. 2, pp. 168-180. 
Tirunillai, S. and Tellis, G.J. (2014), "Mining marketing meaning from online chatter: strategic brand analysis of big data using latent dirichlet allocation", Journal of Marketing Research, Vol. 51 No. 4, pp. 463-479.

Van Laer, T., Edson Escalas, J., Ludwig, S. and Van Den Hende, E.A. (2018), "What happens in Vegas stays on TripAdvisor? A theory and technique to understand narrativity in consumer reviews", Journal of Consumer Research, Vol. 46 No. 2, pp. 267-285.

Wilson, J.H. and Daugherty, P.R. (2018), "Collaborative intelligence: humans and AI are joining forces", Harvard Business Review, Vol. 96 No. 4, pp. 114-123.

Yassine, M. and Haij, H. (2010), "A framework for emotion mining from text in online social networks", presented at the IEEE International Conference on Data Mining Workshops, IEEE, pp. 1136-1142.

Zamith, R. and Lewis, S.C. (2015), "Content analysis and the algorithmic coder: what computational social science means for traditional modes of media analysis", The ANNALS of the American Academy of Political and Social Science, Vol. 659 No. 1, pp. 307-318.

Zavestoski, S. (2002), "The social-psychological bases of anticonsumption attitudes", Psychology and Marketing, Vol. 19 No. 2, pp. 149-165.

Zhu, W.-D.J., Foyle, B., Gagné, D., Gupta, V., Magdalen, J., Mundi, A.S., Nasukawa, T., Paulis, M., Singer, J. and Triska, M. (2014), IBM Watson Content Analytics: Discovering Actionable Insight from Your Content, retrieved from http://www.redbooks.ibm.com/redbooks/pdfs/sg247877.pdf. 